\title{
Triple versus dual antiplatelet therapy for coronary heart disease patients undergoing percutaneous coronary intervention: A meta-analysis
}

\author{
HONG ZHOU, XIAO-LING FENG，HONG-YING ZHANG，FEI-FEI XU and JIE ZHU
}

Department of Emergency, The Fourth Affiliated Hospital of China Medical University, Shenyang, Liaoning 110032, P.R. China

Received April 4, 2013; Accepted July 16, 2013

DOI: $10.3892 /$ etm.2013.1238

\begin{abstract}
Coronary heart disease (CHD) is the leading cause of mortality worldwide. Previous studies have suggested that cilostazol-based triple antiplatelet therapy (TAT) may be more effective than conventional dual antiplatelet therapy (DAT) at improving the clinical outcomes of patients with CHD undergoing percutaneous coronary intervention (PCI). However, individually published results are inconclusive. The present meta-analysis evaluated controlled clinical studies to compare the clinical outcomes between TAT and DAT in patients with CHD undergoing PCI. Ten controlled clinical studies were included, with a total of 7,670 patients with CHD undergoing PCI. The total number included 3,925 patients treated with DAT (aspirin and clopidogrel) and 3745 patients treated with TAT (addition of cilostazol to DAT). The crude odds ratio (OR) with a $95 \%$ confidence interval (CI) was calculated with either the fixed or random effects model. The meta-analysis results indicated that patients in the TAT group had a significantly lower rate of restenosis compared with that of the DAT group $(\mathrm{OR}=0.59,95 \% \mathrm{CI}$ : 0.45-0.77; $\mathrm{P}<0.001)$. The rate of major adverse cardiac events (MACE) and target lesion revascularization (TLR) in the TAT group were significantly lower compared with those in the DAT group (MACE: $\mathrm{OR}=0.69,95 \% \mathrm{CI}: 0.56-0.85, \mathrm{P}<0.001$; TLR: $\mathrm{OR}=0.61$, 95\% CI: $0.43-0.88, \mathrm{P}=0.008)$. However, no significant differences between the TAT and DAT groups in terms of mortality rate, myocardial infarction, target vessel revascularization and stent thrombosis were observed. In conclusion, the results of the present meta-analysis indicated that the efficacy and safety of cilostazol-based TAT therapy is greater than that of conventional DAT therapy for patients with CHD undergoing PCI.
\end{abstract}

Correspondence to: Dr Jie Zhu, Department of Emergency, The Fourth Affiliated Hospital of China Medical University, 4 Chongshan East Road, Huanggu, Shenyang, Liaoning 110032, P.R. China

E-mail: cmu4h_zj@126.com

Key words: cilostazol, antiplatelet therapy, percutaneous coronary intervention, coronary heart disease, meta-analysis

\section{Introduction}

Percutaneous coronary intervention (PCI), also known as coronary angioplasty, is a non-surgical method used to treat narrowed coronary arteries that supply the heart muscle with blood (1). PCI has been clinically applied for almost 30 years and has become one of the main treatments for coronary heart disease (CHD) (2). PCI with coronary stent implantation has been demonstrated to consistently reduce the symptoms of coronary artery disease and decrease cardiac ischemia; however, PCI has not been shown to reduce mortality rates in large clinical trials (3). The implantable vascular stents used during PCI procedures appear to increase the risk of coronary artery intimal injury and platelet activation, and may thereby increase the risk of thrombosis (4). This is significant, as the 1-year mortality rate of patients with myocardial infarction (MI) induced by thrombotic diseases is $\sim 15.8 \%$ (5). Therefore, antiplatelet therapy has become the focus of basic interventional cardiology studies and has received increased clinical attention in the last decade (6).

Dual antiplatelet therapy (DAT; aspirin and clopidogrel) is a mainstay of medical treatment following PCI, which reduces the risk of occurrence of stent thrombosis. However, studies have demonstrated that aspirin and clopidogrel resistance are occurring clinically with potentially severe consequences, including recurrent MI, stroke or mortality (7). Therefore, triple antiplatelet therapy (TAT; addition of cilostazol to DAT) has been increasingly studied (8). Cilostazol is a selective phosphodiesterase-3 inhibitor that is commonly used as a vasodilator with antiplatelet activity in patients with peripheral arterial disease (9). Adding cilostazol to aspirin and clopidogrel regimens may provide a more effective suppression of platelet P-selectin expression in patients with relatively high platelet activity (10). In addition, TAT has also been demonstrated to have anti-inflammatory, -apoptotic and -proliferative effects, as demonstrated by its reduction of intimal hyperplasia and restenosis following balloon angioplasty and stent implantation (11). Registry data have further identified that TAT reduces the rate of restenosis, incidence of clinical events and stent thrombosis, compared with DAT (12). However, controlled clinical studies that have examined the benefits of adding cilostazol to DAT in patients with CHD undergoing PCI with coronary stent implantation have obtained conflicted or inconclusive results. Therefore, the present meta-analysis aimed to 
compare differences in the clinical outcomes between DAT and TAT in patients with CHD undergoing PCI.

\section{Methods}

Literature search strategy. Relevant studies published prior to March 1, 2013, were identified by searches in Pubmed, Embase, Web of Science and Chinese BioMedical databases using the following terms: ('coronary disease' or 'coronary diseases' or 'disease, coronary' or 'coronary heart disease' or 'heart disease, coronary' or 'stents' or 'stent' or 'drug-eluting stents' or 'bare metal stent' or 'percutaneous coronary intervention', and 'triple antiplatelet therapy' or 'dual antiplatelet therapy' or 'TAT' or 'DAT' or 'cilostazol' or 'clopidogrel' or 'aspirin'). References from eligible articles or textbooks were also reviewed to find further potential sources. Disagreements were resolved through discussion between authors.

Inclusion and exclusion criteria. Studies included in the meta-analysis were required to meet the following criteria: i) controlled clinical study focusing on the differences in the clinical outcomes between TAT and DAT in patients with CHD undergoing PCI with coronary stent implantation; ii) patients in the DAT group were treated with aspirin and clopidogrel and patients in the TAT group were treated with cilostazol in addition to aspirin and clopidogrel; iii) the follow-up period was $>1$ month and iv) published data of the clinical outcomes were sufficient. Studies were excluded from the meta-analysis when they were: i) not controlled clinical studies relevant to TAT and DAT in patients with CHD undergoing PCI with coronary stent implantation; ii) duplicates of previous publications; iii) based on incomplete data; or iv) meta-analyses, letters, reviews or editorial articles. When more than one study by the same author and using the same case series was published, either the study with the largest sample size or the most recently published study was included.

Data extraction. Using a standardized form, data from published studies were extracted independently by two authors. For each study, the following characteristics and numbers were collected: the authors, year of publication, country, ethnicity, study design, number of subjects, follow-up period, antiplatelet drug and dose, rate of restenosis and clinical events [major adverse cardiac events (MACE) and stent thrombosis]. MACE included mortality rate, MI, target lesion revascularization (TLR) and target vessel revascularization (TVR). In cases of conflicting evaluations, two authors discussed the issue in order to meet a consensus; if no agreement was reached, a third author decided.

Statistical analysis. The crude odds ratio (OR) with 95\% confidence interval (CI) was calculated. The statistical significance of the pooled OR was examined by the Z-test. Interstudy variations and heterogeneities were estimated using Cochran's Q-statistic with $\mathrm{P}<0.05$ indicating a statistically significant heterogeneity $(13,14)$. The present meta-analysis also quantified the effect of heterogeneity by using the $\mathrm{I}^{2}$ index (range, $0-100 \%$ ), which represents the proportion of interstudy variability attributed to heterogeneity, rather than to chance. When a significant Q-test

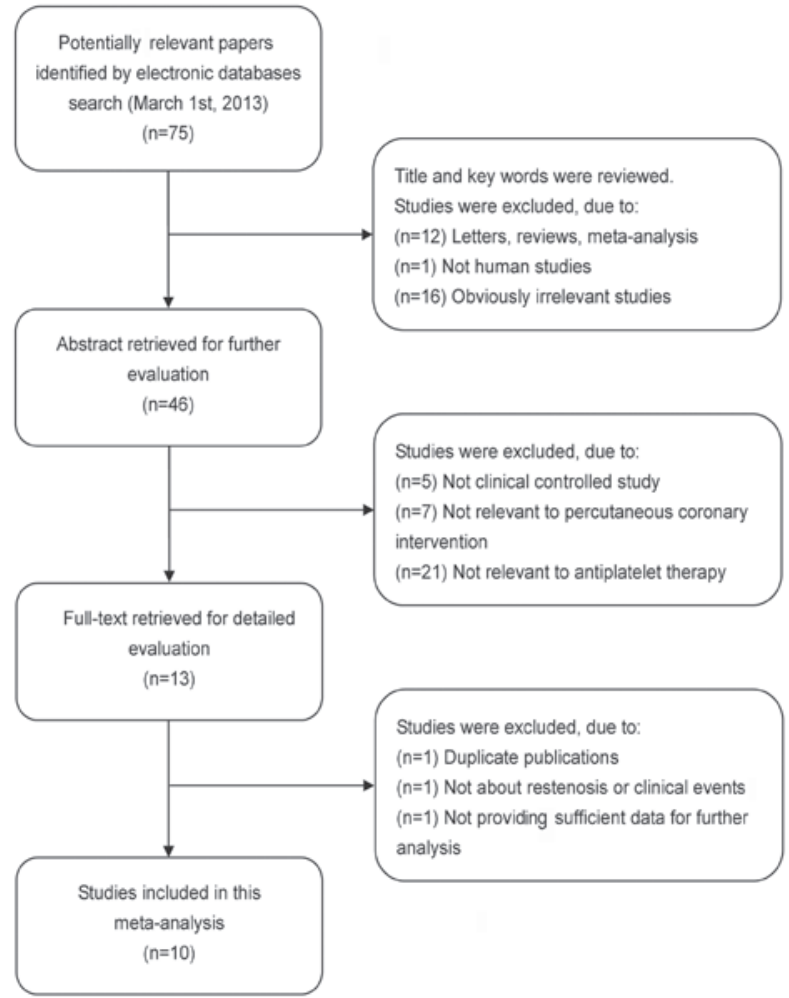

Figure 1. Flow chart of literature search and study selection.

$(\mathrm{P}<0.05)$ or an $\mathrm{I}^{2}$ index of $>50 \%$ was obtained, indicating that heterogeneity existed among studies, the random effects model using the DerSimonian and Laird method (15) was conducted. However, when the Q-test was not significant $(\mathrm{P}>0.05)$ or the $\mathrm{I}^{2}$ index was $<50 \%$, the fixed effects model using the Mantel-Haenszel method (16) was used. To explore sources of heterogeneity, subgroup analysis was performed by follow-up periods. A sensitivity analysis was performed by omitting each study in turn, to assess the quality and consistency of the results. Begg's funnel plot was used to detect publication bias. Egger's linear regression test, which measures funnel plot asymmetry using a natural logarithm scale of OR, was also used to evaluate publication bias (17). To ensure the reliability and accuracy of the results, two authors examined the data independently using statistical software programs and obtained identical results. The $\mathrm{P}$-values were two-sided and $\mathrm{P}<0.05$ was considered to indicate a statistically significant difference. All analyses were calculated using Stata software, version 12.0 (StataCorp LP, College Station, TX, USA).

\section{Results}

Characteristics of the included studies. In the present meta-analysis, according to the inclusion criteria, 10 controlled clinical studies were included $(8,12,18-25)$ and 65 were excluded. The publication year of the included studies ranged from 2005 to 2011. The flow chart of study selection is shown in Fig. 1. A total of 7,670 patients with CHD undergoing PCI were involved in the present meta-analysis, including 3,925 patients treated with DAT (aspirin and clopidogrel) and 3,745 patients treated with TAT (addition of cilostazol to 


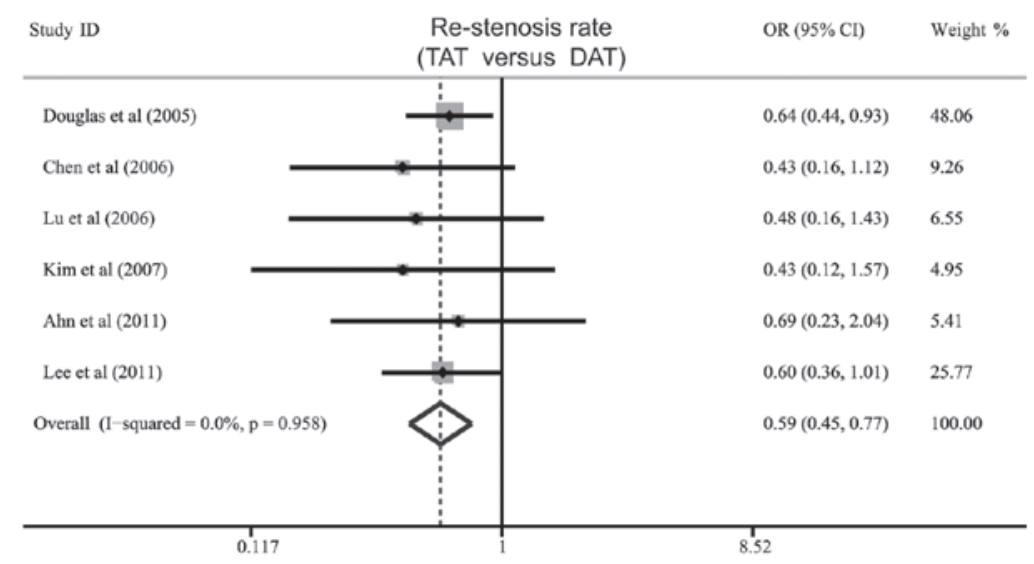

Figure 2. Forest plot of crude OR for differences in the restenosis rate between TAT and DAT. OR, odds ratio; TAT, triple antiplatelet therapy; DAT, dual antiplatelet therapy; CI, confidence interval.

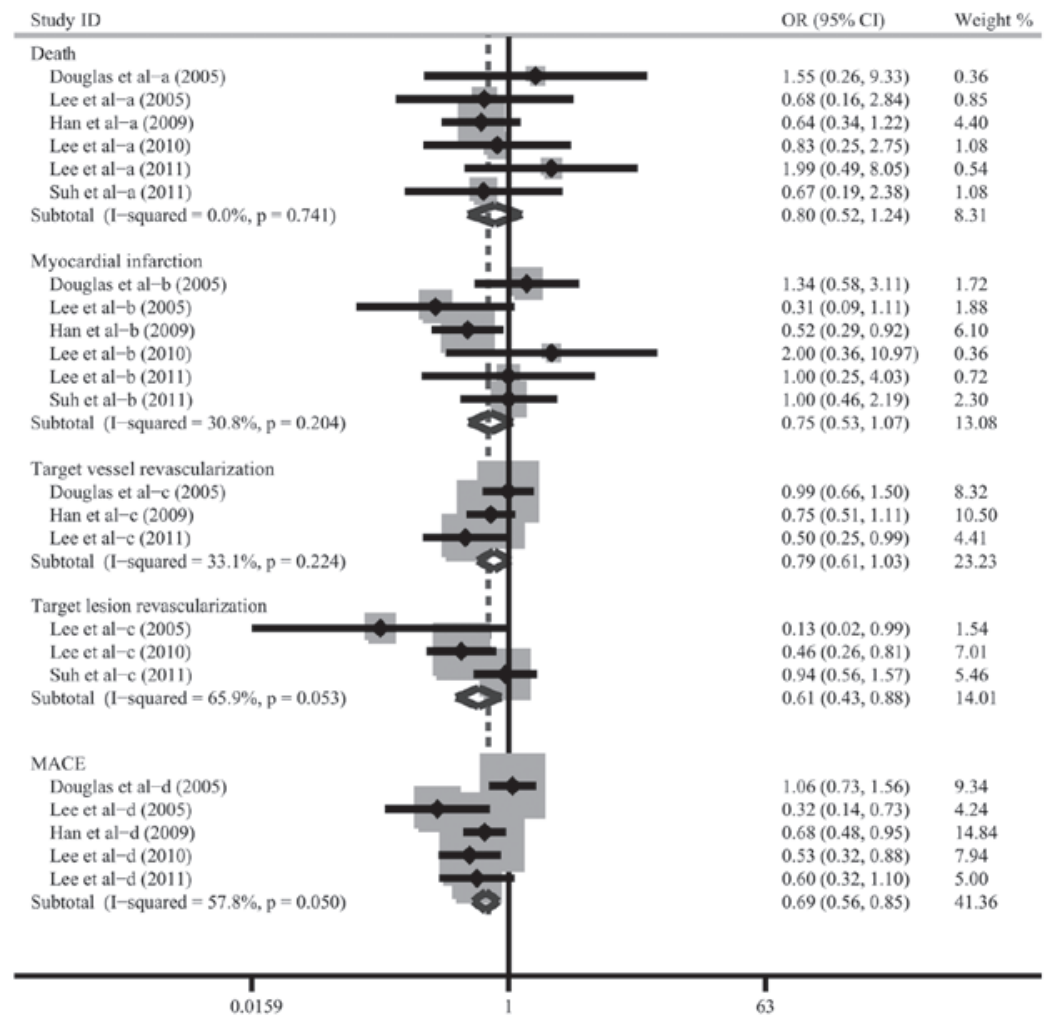

Figure 3. Forest plot of crude OR for differences in the rates of MACE between TAT and DAT. MACE, major adverse cardiac events; CI, confidence interval; OR, odds ratio; TAT, triple antiplatelet therapy; DAT, dual antiplatelet therapy.

DAT). The doses of aspirin ranged from 100 to $200 \mathrm{mg} / \mathrm{day}$, those of cilostazol ranged from 100 to $200 \mathrm{mg} /$ day and the dose of clopidogrel was $75 \mathrm{mg} /$ day. The main characteristics of the eligible studies are listed in Table I.

Quantitative data synthesis. Six studies identified a difference in the rate of restenosis between TAT and DAT for patients with CHD undergoing PCI. The heterogeneity was not significant $\left(\mathrm{P}=0.958, \mathrm{I}^{2}=0 \%\right)$, therefore, the fixed effects model was used. The meta-analysis results showed that patients in the TAT group had a significantly lower rate of restenosis than those in the DAT group (OR $=0.59,95 \%$ CI: 0.45-0.77, P<0.001; Fig. 2).
The differences in the clinical events between TAT and DAT were compared in six studies. As no heterogeneity was identified, the fixed effects model was used. The meta-analysis results indicated that the rates of MACE and TLR in the TAT group were significantly lower compared with those in the DAT group (MACE: OR=0.69, 95\% CI: 0.56-0.85, P<0.001; TLR: OR=0.61, 95\% CI: 0.43-0.88, $\mathrm{P}=0.008)$. However, no significant differences were indicated in mortality rates $(\mathrm{OR}=0.80,95 \% \mathrm{CI}: 0.52-1.24, \mathrm{P}=0.319)$, MI (OR=0.75, 95\% CI: 0.53-1.07, $\mathrm{P}=0.109)$ and TVR $(\mathrm{OR}=0.79,95 \%$ CI: $0.61-1.03, \mathrm{P}=0.077)$ between the TAT and DAT groups (Fig. 3). Additionally, no significant difference was identified in the rate of stent thrombosis between 


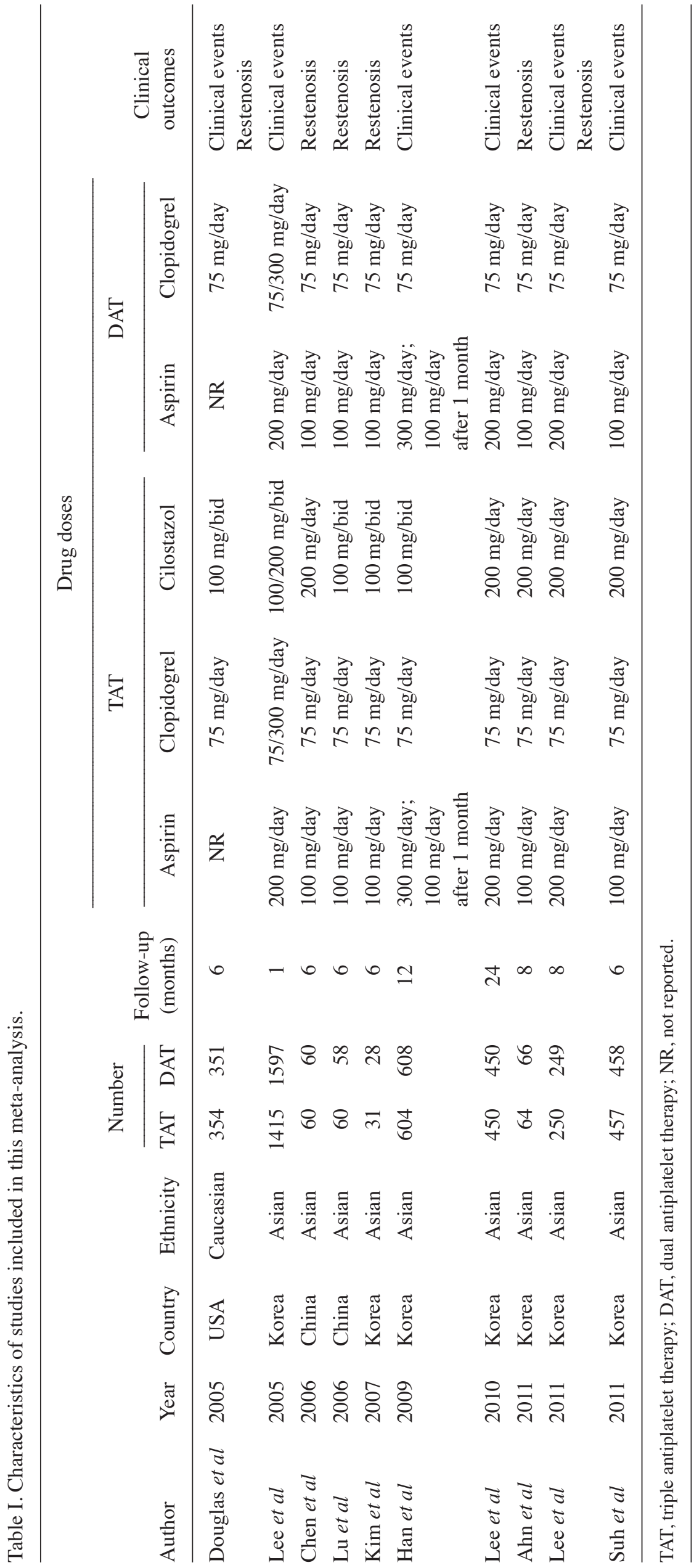




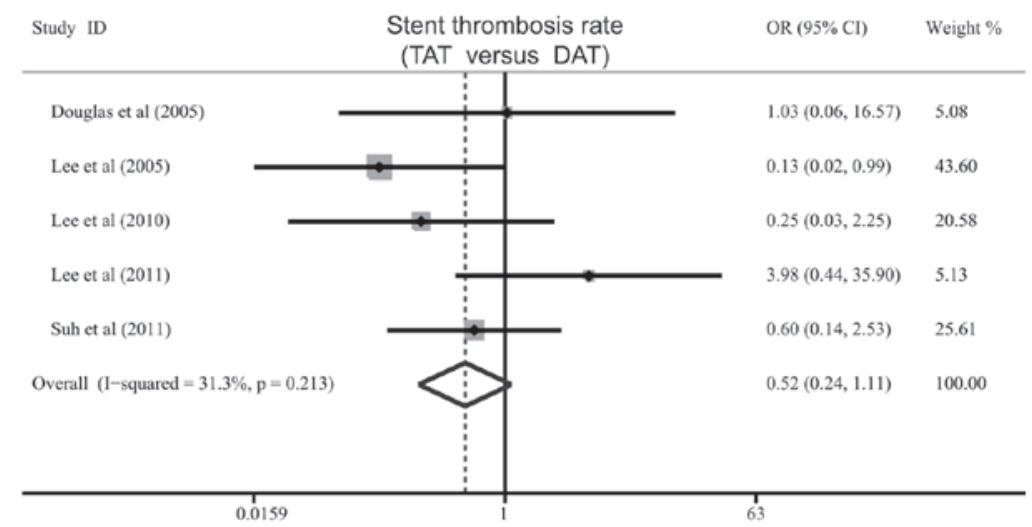

Figure 4. Forest plot of crude OR for differences in the rate of stent thrombosis between TAT and DAT. OR, odds ratio; TAT, triple antiplatelet therapy; DAT, dual antiplatelet therapy; $\mathrm{CI}$, confidence interval.

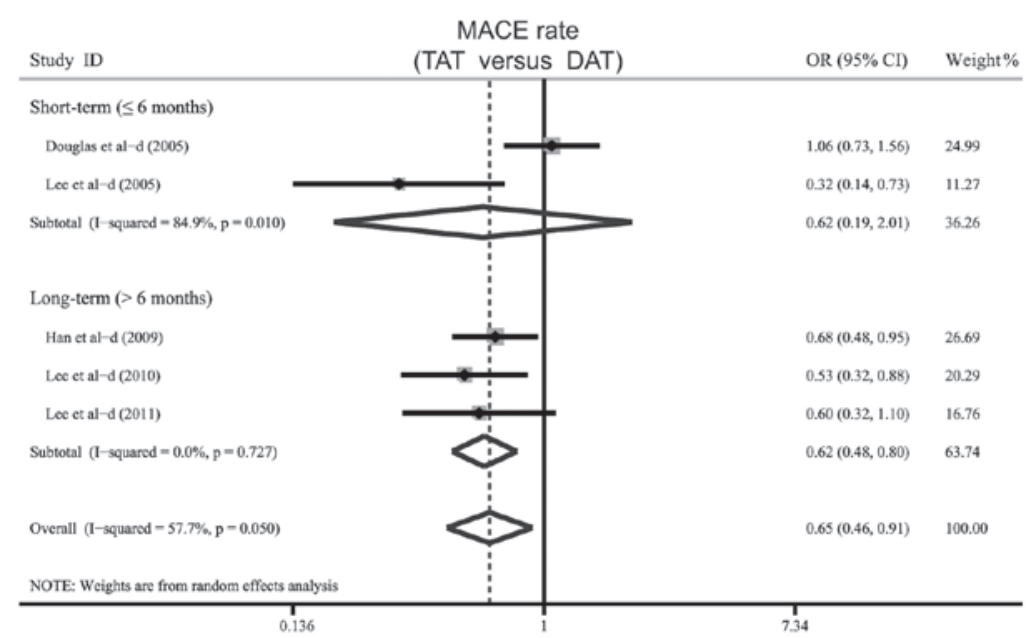

Figure 5. Subgroup analysis by follow-up periods of crude OR for the rate of MACE between TAT and DAT. OR, odds ratio; MACE, major adverse cardia events; TAT, triple antiplatelet therapy; DAT, dual antiplatelet therapy; CI, confidence interval.

the TAT and DAT groups (OR=0.52, 95\% CI: 0.24-1.11, $\mathrm{P}=0.091$; Fig. 4).

When examining the potential factors that may have impacted the results, a further subgroup analysis was performed based on the follow-up periods. The results indicated a significant difference in the rate of MACE between TAT and DAT in the long-term ( $>6$ months) follow-up subgroups $(\mathrm{OR}=0.62,95 \%$ CI: $0.48-0.80, \mathrm{P}<0.001)$. However, no significant difference in the rate of MACE was indicated in the short-term ( $\leq 6$ months) follow-up subgroups ( $\mathrm{OR}=0.62$, 95\% CI: 0.19-2.01, P=0.421; Fig. 5).

Sensitivity analysis and publication bias. Sensitivity analysis was performed to assess the influence of each individual study on the pooled ORs by omitting individual studies. Analysis of the results suggested that no individual study significantly affected the pooled ORs of the rate of restenosis and MACE (Fig. 6), indicating a statistically robust result.

Publication bias exists to the extent that the available research results are not representative of all research results. Begg's funnel plot and Egger's linear regression test were performed to assess the publication bias of the included studies. The shapes of the funnel plots did not demonstrate any obvious asymmetry (Fig. 7). Egger's test also showed that there was no statistical indication of publication bias for the rates of restenosis and MACE (restenosis: $\mathrm{t}=-2.04, \mathrm{P}=0.111$; MACE: $\mathrm{t}=-1.76, \mathrm{P}=0.177)$.

\section{Discussion}

The clinical application of PCI with coronary stent implantation has markedly increased, which has significantly improved the outcomes in patients with CHD (26). However, serious complications following implantation of coronary stents remain a significant clinical problem and may result in failure of PCI to provide long-term benefits. Over the past decade, numerous studies have indicated that the use of adjunctive antiplatelet agents has resulted in decreased stent-associated thrombosis and is important in improving the final outcomes of PCI (27). Two meta-analyses by Tamhane et al (11) and Singh et al (28) also indicated that patients treated with TAT exhibited a lower restenosis rate than those treated with DAT $(11,28)$. However, these meta-analyses did not provide reliable results demonstrating the differences in the clinical outcomes between TAT and DAT for patients with CHD undergoing PCI with coronary stent implantation. Therefore, in view of the conflicting results 
A

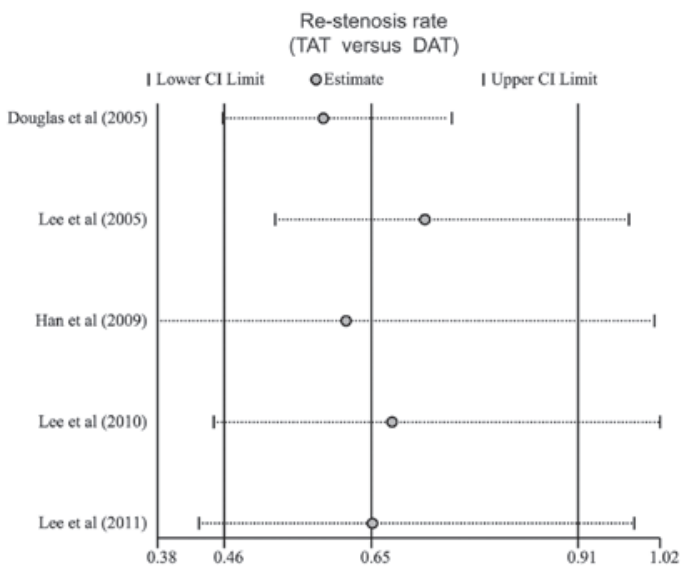

B

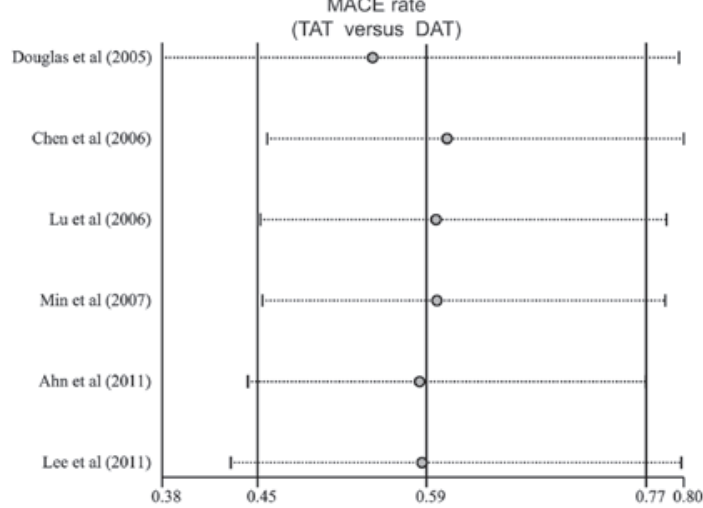

Figure 6. Sensitivity analysis of the summary odds ratio coefficients on the rates of restenosis and MACE between TAT and DAT. (A) Restenosis rate; (B) MACE rate. Results were computed by omitting each study in turn. Meta-analysis random-effects estimates (exponential form) were used. The two ends of the dotted lines represent the 95\% CI. MACE, major adverse cardiac events; TAT, triple antiplatelet therapy; DAT, dual antiplatelet therpay; CI, confidence interval.

from previous studies and the insufficient statistical power of the two previous meta-analyses, the present meta-analysis was performed in order to update previous meta-analyses and provide a comprehensive conclusion.

In the present meta-analysis, 10 clinical studies were included with a total of 7,670 patients with CHD undergoing PCI with coronary stent implantation, including 3,745 patients in the TAT group and 3,925 patients in the DAT group. The predominant finding of this meta-analysis was that the rates of restenosis, MACE and TLR in the TAT group were significantly lower compared with those of the DAT group. Furthermore, when the follow-up periods were stratified into short-term ( $\leq 6$ months) and long-term ( $>6$ months) subgroups, it was noteworthy that a significant difference in the rate of MACE between TAT and DAT was only observed in the long-term follow-up subgroup. The substantial improvement due to the addition of cilostazol to DAT identified by the current meta-analysis is consistent with the findings of several previous studies. This is due to cilostazol inhibiting the progression of carotid intima-media thickness and thus producing an additional anti-proliferative effect, regardless of the fact that DAT itself is a potent therapy $(23,29-31)$. Notably, the significantly reduced rate of MACE in the long-term follow-up subgroup is inconsistent with the findings of two previous meta-analyses, which indicated that there was no
A
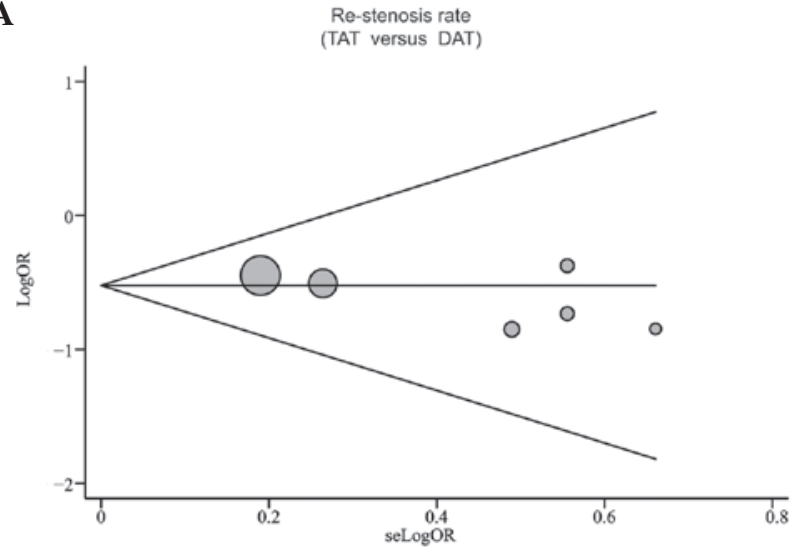

B

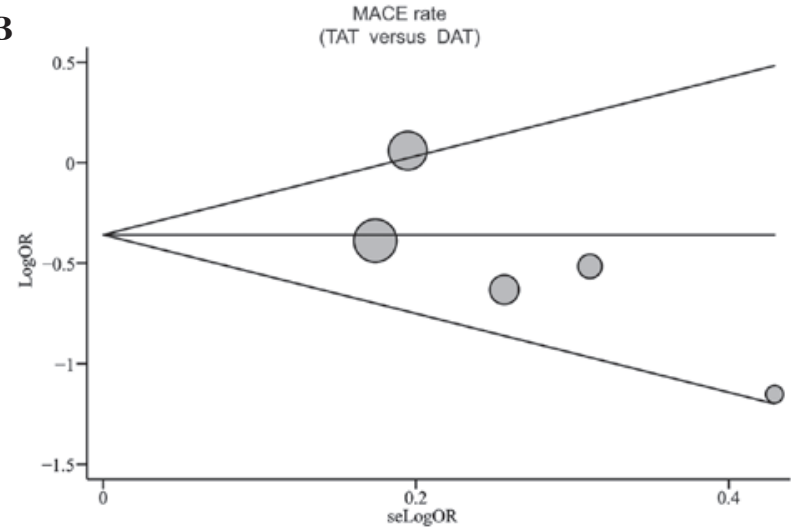

Figure 7. Begg's funnel plot of publication bias in a selection of studies on the rates of restenosis and MACE between TAT and DAT. (A) Restenosis rate; (B) MACE rate. Each point represents a separate study for the indicated association. Log (OR), natural logarithm of OR. Horizontal line, mean magnitude of the effect. MACE, major adverse cardiac events; TAT, triple antiplatelet therapy; DAT, dual antiplatelet therpay; OR, odds ratio.

difference in the clinical outcomes of MACE between DAT and TAT treatment groups $(11,28)$. A potential reason for the discrepancy may be the limited number of included studies. The controversial results may also be caused by the high degree of heterogeneity in the different follow-up periods. In the present meta-analysis, no significant differences in the rates of mortality, MI and TVR were observed between TAT and DAT for patients with CHD undergoing PCI. Additionally, no significant difference in the rate of stent thrombosis between TAT and DAT was determined, which is in accordance with a previous meta-analysis (32). These results suggest that TAT may be no different from DAT in terms of its effectiveness as an adjunctive therapy to coronary stents and as a preventative measure against stent-associated thrombosis. This is regardless of the different antiplatelet mechanisms of TAT, which may result in suppression of platelet aggregation (32). The precise effect of adding cilostazol to DAT on the incidence rates of various clinical events requires further study before conclusions regarding these outcomes may be made.

Similar to previous meta-analyses, the present meta-analysis also has certain limitations. The sample size was relatively small and may not have provided sufficient statistical power to estimate the differences in the clinical outcomes between TAT and DAT for patients with CHD undergoing PCI. A meta-analysis, as a type of retrospective study, may encounter recall or selection bias, which may influence the reliability of the results. A 
further limitation, was that each pretreatment regimen was not consistent and the doses of aspirin or cilostazol were variable. Moreover, the type of stent used in each patient was not consistent and there may have been differences with regard to the effectiveness of the antiplatelet agents. Although all participants in each study were required to meet similar inclusion criteria, there may have been potential factors that were not taken into account that may have influenced the results. Therefore, the results of this meta-analysis should be interpreted with caution, owing to the potential heterogeneity among trials.

In conclusion, the present meta-analysis suggests that the efficacy and safety of cilostazol-based TAT may be greater than that of conventional DAT for treating patients with CHD undergoing PCI. However, additional well-designed and randomized controlled trials are required to further investigate and clarify the differences in the clinical outcomes between TAT and DAT for patients with CHD.

\section{References}

1. Aradi D, Komócsi A, Price MJ, et al: Efficacy and safety of intensified antiplatelet therapy on the basis of platelet reactivity testing in patients after percutaneous coronary intervention: Systematic review and meta-analysis. Int J Cardiol: Jun 15, 2012 (Epub ahead of print)

2. Meryon I, Patel N, Millane T and Varma C: Normal coronary angiography and primary percutaneous coronary intervention for ST elevation myocardial infarction: a literature review and audit findings. Int J Clin Pract 64: 1245-1251, 2010.

3. Mercado N and Serruys PW: A meta-analytical approach for the treatment of in-stent restenosis. Eur Heart J 24: 217-218, 2003.

4. Meier B, Bachmann D and Lüscher T: 25 years of coronary angioplasty: almost a fairy tale. Lancet 361: 527, 2003.

5. Mrdovic I, Savic L, Lasica R, et al: Usefulness of the RISK-PCI score to predict stent thrombosis in patients treated with primary percutaneous coronary intervention for ST-segment elevation myocardial infarction: a substudy of the RISK-PCI trial. Heart Vessels 28: 424-433, 2013.

6. Gurbel PA and Tantry US: Aspirin and clopidogrel resistance: consideration and management. J Interv Cardiol 19: 439-448, 2006.

7. Takahashi S, Kaneda H, Tanaka S, et al: Late angiographic stent thrombosis after sirolimus-eluting stent implantation. Circ J 71: 226-228, 2007.

8. Suh JW, Lee SP, Park KW, et al: Multicenter randomized trial evaluating the efficacy of cilostazol on ischemic vascular complications after drug-eluting stent implantation for coronary heart disease: results of the CILON-T (influence of CILostazol-based triple antiplatelet therapy $\mathrm{ON}$ ischemic complication after drug-eluting stenT implantation) trial. J Am Coll Cardiol 57: 280-289, 2011.

9. Regensteiner JG and Stewart KJ: Established and evolving medical therapies for claudication in patients with peripheral arterial disease. Nat Clin Pract Cardiovasc Med 3: 604-610, 2006.

10. Jeong YH, Lee SW, Choi BR, et al: Randomized comparison of adjunctive cilostazol versus high maintenance dose clopidogre in patients with high post-treatment platelet reactivity: results of the ACCEL-RESISTANCE (Adjunctive Cilostazol Versus High Maintenance Dose Clopidogrel in Patients With Clopidogrel Resistance) randomized study. J Am Coll Cardiol 53: 1101-1109, 2009.

11. Tamhane U, Meier P, Chetcuti S, et al: Efficacy of cilostazol in reducing restenosis in patients undergoing contemporary stent based PCI: a meta-analysis of randomised controlled trials. EuroIntervention 5: 384-393, 2009.

12. Lee SW, Park SW, Hong MK, et al: Triple versus dual antiplatelet therapy after coronary stenting: impact on stent thrombosis. J Am Coll Cardiol 46: 1833-1837, 2005.

13. Higgins JP and Thompson SG: Quantifying heterogeneity in a meta-analysis. Stat Med 21: 1539-1558, 2002.

14. Zintzaras E and Ioannidis JP: Heterogeneity testing in meta-analysis of genome searches. Genet Epidemiol 28: 123-137, 2005.
15. DerSimonian R and Kacker R: Random-effects model for metaanalysis of clinical trials: an update. Contemp Clin Trials 28: 105-114, 2007.

16. Mantel N and Haenszel W: Statistical aspects of the analysis of data from retrospective studies of disease. J Natl Cancer Inst 22: 719-748, 1959.

17. Peters JL, Sutton AJ, Jones DR, Abrams KR and Rushton L: Comparison of two methods to detect publication bias in meta-analysis. JAMA 295: 676-680, 2006.

18. Ahn CM, Hong SJ, Park JH, Kim JS and Lim DS: Cilostazol reduces the progression of carotid intima-media thickness without increasing the risk of bleeding in patients with acute coronary syndrome during a 2-year follow-up. Heart Vessels 26: 502-510, 2011.

19. Chen YD, Lu YL, Jin ZN, Yuan F and Lu SZ: A prospective randomized antiplatelet trial of cilostazol versus clopidogrel in patients with bare metal stent. Chin Med J (Engl) 119: 360-366, 2006.

20. Douglas JS Jr, Holmes DR Jr, Kereiakes DJ, et al: Coronary stent restenosis in patients treated with cilostazol. Circulation 112: 2826-2832, 2005.

21. Han Y, Li Y, Wang S, et al: Cilostazol in addition to aspirin and clopidogrel improves long-term outcomes after percutaneous coronary intervention in patients with acute coronary syndromes: a randomized, controlled study. Am Heart J 157: 733-739, 2009.

22. Kim JY, Lee K, Shin M, et al: Cilostazol could ameliorate platelet responsiveness to clopidogrel in patients undergoing primary percutaneous coronary intervention. Circ J 71: 1867-1872, 2007.

23. Lee SW, Chun KJ, Park SW, et al: Comparison of triple antiplatelet therapy and dual antiplatelet therapy in patients at high risk of restenosis after drug-eluting stent implantation (from the DECLARE-DIABETES and -LONG Trials). Am J Cardiol 105: 168-173, 2010.

24. Lee SW, Park SW, Kim YH, et al: A randomized, double-blind, multicenter comparison study of triple antiplatelet therapy with dual antiplatelet therapy to reduce restenosis after drug-eluting stent implantation in long coronary lesions: results from the DECLARE-LONG II (Drug-Eluting Stenting Followed by Cilostazol Treatment Reduces Late Restenosis in Patients with Long Coronary Lesions) trial. J Am Coll Cardiol 57: 1264-1270, 2011.

25. Lu YL, Chen YZ, Lv SZ, Pan WZ and Liu X: Cilostazol prevention in elderly coronary heart disease patients with coronary bare metal stent restenosis after effect. Chin J Geriatr 25: 537-538, 2006 (In Chinese)

26. Schleinitz MD, Olkin I and Heidenreich PA: Cilostazol, clopidogrel or ticlopidine to prevent sub-acute stent thrombosis: a meta-analysis of randomized trials. Am Heart J 148: 990-997, 2004.

27. Smith SC Jr., Dove JT, Jacobs AK, et al: ACC/AHA guidelines for percutaneous coronary intervention (revision of the 1993 PTCA guidelines)-executive summary: a report of the American College of Cardiology/American Heart Association task force on practice guidelines (Committee to revise the 1993 guidelines for percutaneous transluminal coronary angioplasty) endorsed by the Society for Cardiac Angiography and Interventions. Circulation 103: 3019-3041, 2001.

28. Singh I, Shafiq N, Pandhi P, et al: Triple antiplatelet therapy vs. dual antiplatelet therapy in patients undergoing percutaneous coronary intervention: an evidence-based approach to answering a clinical query. Br J Clin Pharmacol 68: 4-13, 2009.

29. Biondi-Zoccai GG, Lotrionte M, Anselmino M, et al: Systematic review and meta-analysis of randomized clinical trials appraising the impact of cilostazol after percutaneous coronary intervention. Am Heart J 155: 1081-1089, 2008.

30. Friedland SN, Eisenberg MJ and Shimony A: Meta-analysis of randomized controlled trials on effect of cilostazol on restenosis rates and outcomes after percutaneous coronary intervention. Am J Cardiol 109: 1397-1404, 2012.

31. Hong SJ, Choi SC, Ahn CM, Park JH, Kim JS and Lim DS: Telmisartan reduces neointima volume and pulse wave velocity 8 months after zotarolimus-eluting stent implantation in hypertensive type 2 diabetic patients. Heart 97: 1425-1432, 2011.

32. Hashiguchi M, Ohno K, Nakazawa R, Kishino S, Mochizuki M and Shiga T: Comparison of cilostazol and ticlopidine for one-month effectiveness and safety after elective coronary stenting. Cardiovasc Drugs Ther 18: 211-217, 2004. 